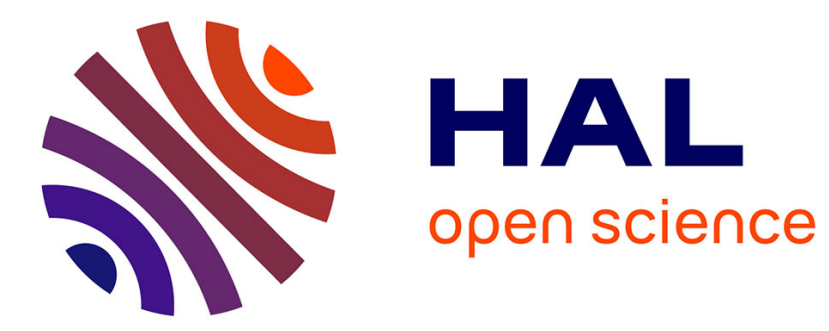

\title{
On Certain Types of Product Set-Labeling of Graphs
}

Sudev Naduvath

\section{To cite this version:}

Sudev Naduvath. On Certain Types of Product Set-Labeling of Graphs. Journal of Combinatorics, Information \& System Sciences, 2018. hal-02263306

\section{HAL Id: hal-02263306 https://hal.science/hal-02263306}

Submitted on 5 Aug 2019

HAL is a multi-disciplinary open access archive for the deposit and dissemination of scientific research documents, whether they are published or not. The documents may come from teaching and research institutions in France or abroad, or from public or private research centers.
L'archive ouverte pluridisciplinaire HAL, est destinée au dépôt et à la diffusion de documents scientifiques de niveau recherche, publiés ou non, émanant des établissements d'enseignement et de recherche français ou étrangers, des laboratoires publics ou privés. 


\title{
On Certain Types of Product Set-Labeling of Graphs
}

\author{
Sudev NAduvath
}

\begin{abstract}
The product set of two sets $A$ and $B$ of integers, denoted by $A * B$, is the set $A * B=$ $\{a b: a \in A, b \in \mathrm{B}\}$. For $X \subseteq \mathbb{N}$, a product set-labeling (PS-labeling) of a graph $\mathrm{G}$ is an injective function $f: V(G) \rightarrow P(X)$ such that the induced function $\mathrm{f}^{*}: V(G) \rightarrow P(X)$ is defined as $f^{*}(u v)=f(u) * f(v) \forall u v \in E(G), f(u) * f(v)$ being the product set of $f(u)$ and $f(v)$. The PS-labeling of a graph can be classified into certain types in two ways: in accordance with the cardinalities of the set-labels and according to the nature of the collection of set-labels of elements of the graph $G$. This paper discusses different types of PS-labeling of graphs.
\end{abstract}

Keywords: Product set-labeling of graphs, GPS-labeling, SPS-labeling, exquisite PS-labeling, TPS-Labeling.

MSC 2010: 05 C78.

\section{INTRODUCTION :}

For the terms and definitions, which are not introduced in this paper, we refer to $[2,4,9]$. Unless stated otherwise, the graphs we consider in this paper are simple, finite, undirected and connected.

A set-labeling or a set-valuation of a graph $G$ is a one to one mapping under which the vertices of $G$ assumes the subsets of a non-empty ground set $X$ as their labels and the edges of $G$ has the labels obtained by some binary operations of the labels of their end vertices (see [1]). A set-labeled graph (or a set-valued graph) is a graph $G$ with a setlabeling defined on it.

Many graph theorists have comprehensively and extensively studied various types of set-valuations of graphs and a significant number of innovative research papers have been

J. of Combinatorics, Information \& System Sciences Vol. 43 (2018), No. 1-4, pages 131-140

(C) Forum for Interdisciplinary Mathematics

Corresponding author email: sudevnk@gmail.com 
published thereafter. Among these, some interesting studies dealt with structural properties and characteristics of such set-valued graphs in a much rigorous manner.

A significant deviation from the typical studies on set-labeled graphs was the introduction of a particular set-labeling namely integer additive set-labeling of graphs (see [3, 6]). In this type, the set-labels of the vertices of graphs are non-empty finite sets of non-negative integers and the set-label of each edge is the sumset of the set-labels of its end vertices (see [3]). Some interesting types of integer additive set-labelings have been done in $[3,6,7,8]$.

Following the studies on integer additive set-labeling of graphs, a new type set-labeling, namely, the product set-labeling (PS-labeling) of graphs has been introduced in [5]. Motivated by these studies, in this paper, we discuss certain types of PS-labeling of graphs, and investigate the structural properties of the graphs which admit the PS-labelings and characteristics of the set-labels of vertices and edges of graphs with respect to these PS-labelings.

\section{PRODUCT SET-LABELING OF GRAPHS}

Let $\mathbb{N}$ be the set of all positive integers and $X \subseteq \mathbb{N}$. The product set-labeling (PS-labeling) of graphs was introduced in [5] as an injective function an injective set-valued function $f: V(G)$ $\rightarrow \mathrm{P}(X)$ such that the induced edge-function $f^{*}: V(G) \rightarrow P(X)$ is defined as $f^{*}(u v)=f(u) *$ $f(v) \forall u v \in E(G)$, where $f(u) * f(v)=\{a b: a \in f(u) ; b \in f(v)\}$, the product set of the setlabels $f(u)$ and $f(v)$. Certain types of PS-labelings have also been discussed in [5].

\subsection{Preliminaries}

A PS-labeling may be called a $k$-uniform PS-labeling if $|f *(u v)|=k$, where $k \in \mathbb{N}$. For $u v \in$ $E(G)$, note that $|f(u)|+|f(v)|-1 \leq|f(u v)| \leq|f *(u v)| \leq|f(u)||f(v)|$. When $|f(u)|+|f(v)|-1=$ $|f *(u v)|$, then both $f(u)$ and $f(v)$ are geometric progressions and the corresponding PS-labeling of $G$ is called a geometric product set-labeling (GPS-labeling). If $|f *(u v)|=|f(u) \| f(v)|$, the corresponding set-labeling of $G$ is called a strong product set-labeling (SPS-labeling) (see [5]).

A necessary and sufficient condition for a PS-labeling $f: V(G) \rightarrow P(X) ; \mathrm{X} \subseteq \mathbb{N}$ has been established in [5] as follows:

Theorem 2.1. [5] A PS-labeling f : $V(G) \rightarrow P(N)$ of a graph $G$ is a GPS-labeling of $G$ if and only if for every edge of $G$, the common ratio of one end vertex is a positive integral

$\overline{\text { Journal of Combinatorics, Information } \& \text { System Sciences }}$ 
power of the common ratio of the other end vertex, where this power is less than or equal to the label size of the end vertex having smaller common ratio.

The following result was on the label size (cardinality of the set-label) of the edges of a graph $G$ which admits a GPS-labeling.

Theorem 2.2. [5] Let $f$ be a GPS-labeling of a graph $G$ and let $u$ and $v$ be two adjacent vertices in $\mathrm{G}$ with the common ratios $r_{u}$ and $r_{v}$ such that $r_{u} \leq r_{v}$. Then, the label size of the edge uv is given by $\left.\left|f^{*}(u v)\right|=|f(u)|+k|f(v)|-1\right)$, where $k$ is the characteristic index of the edge $u v$.

If the context is clear, the common ratio of the set-label of an element of $\mathrm{G}$ may be called the common ratio of that element. The characteristic index of an edge $e=u v$ of a product set-labeled graph G is the number $k \geq 1$, such that $r_{v}=\left(r_{u}\right)^{k}$, where $r_{u}$ and $r_{v}$ are the common ratios of the vertices $u$ and $v$ (or equivalently, the common ratios of $u$ and $v$ ) respectively. An isogeometric product set-labeling (IPS-labeling) is a geometric set-labeling of $G$, where the characteristic index of all edges of $G$ are the same.

It can be easily verified that every graph admits a GPS-labeling (and IPS- labeling) for some suitable choices of the ground set $X$.

The following theorem discusses a necessary and suficient condition for a GPS- labeling $f$ of a graph $G$ to be a uniform PS-labeling.

Theorem 2.3. A GPS-labeling of a connected graph $G$ is a uniform PS-labeling of $G$ if and only if $G$ is a bipartite graph or all its vertices have the same label size.

Proof. Let $f$ be a GPS-labeling of a given graph $G$. First assume that $|f(v)|=t \forall v \in V$ $(G)$. Then, by Theorem 2.2, every edge of $G$ has the set-label $\ell=t+k(\mathrm{t}-1)$, where $k$ is the characteristic index of edges in $G$. Therefore, $f$ is an $\ell$-uniform PS-labeling of $G$.

Next, assume that $G$ is bipartite with the bipartition $\left(V_{1} ; V_{2}\right)$. Then, for suitable choice of the ground set $X$, we can label all vertices in $V_{1}$ by distinct geometric progressions of the same size, say $n_{1}$ and the common ratio, say $r_{1}$ and can label all vertices of $V_{2}$ by distinct geometric progressions of the same size, say $n_{2}$ and common ratio, say $r_{2}$ such that $r_{1} \leq r_{2}$. Then, by Theorem 2.2, every edge of $G$ has label size $\ell=n_{1}+k\left(n_{2}-1\right)$, where $k \leq n_{1}$ is the characteristic index of the edges in $G$. Hence, this GPS-labeling of $G$ is an $l$-uniform PS-labeling.

Conversely, let $f$ be an $\ell$-uniform GPS-labeling of $G$. If $|f(v)|=\frac{\ell+1}{2}$ for all $v \in V(G)$, then the proof is complete. Hence, assume that not all vertices of $G$ have the same label size. Let 
$V_{1}$ be a (maximal) independent set of $\mathrm{G}$ and label all vertices of $V_{1}$ by geometric progressions with common ratio $r$ and label size $\mathrm{m}$. Then, every vertex $w_{j}$ in $V_{2}$ which is adjacent to any vertex $\mathrm{V}_{\mathrm{i}}$ in $V_{1}$, must be labeled by a geometric progression with common ratio $r_{j}$ and size $\lim _{\mathrm{i} \rightarrow \infty}$ +1 such that $r=k_{i}+1_{r i}$, where $k_{i}$ is the characteristic index of the edge $v_{i} w_{j}$. Since $G$ is $\ell$ uniform, it can be observed that these vertices in $V_{2}$ can be adjacent only to the vertices in $V_{1}$ and not among them. Therefore, $\left(V_{1}, V_{2}\right)$ is a bipartition of $G$. This completes the proof.

Let us call an $\ell$-uniform set-labeled graph whose vertices also have the same label size $m$, an $(m, \ell)$-uniform set-labeled graph. Then, we have

Theorem 2.4. Let $f$ be a GPS-labeling of $G$ whose vertices have the same label size, say $\mathrm{m}$, and let $\ell$ be an even integer. Then, $\mathrm{f}$ is an $\ell$-uniform PS-labeling of $G$ if and only if both $\mathrm{m}$ and the characteristic index $k$ of all edges of $G$ are simultaneously even.

Proof. Since $f$ is a uniform GPS-labeling of $G$, with $f(v)=m$ for all $v \in V(G)$, we have $f(e)=m+k(m-1) \forall e \in E(G)$. Then, the result follows from the following table.

\begin{tabular}{|l|c|c|}
\hline & $m$ is even & $m$ is odd \\
\hline$k$ is even & $m+\mathrm{k}(m-1)$ is even & $m+k(m-1)$ is odd \\
\hline$k$ is odd & $m+k(m-1)$ is odd & $m+k(m-1)$ is odd \\
\hline
\end{tabular}

The result given below is a necessary and suficient condition for a strong GPS- labeling $f$ of a graph $G$ to be a uniform PS-labeling.

Theorem 2.5. A strong GPS-labeling f of a given connected graph $G$ is an $\ell$-uniform PSlabeling of $G$ if and only if either $G$ is bipartite or every vertex of $G$ has the same label size $m$, where $m_{2}=\ell$.

Proof. Let $f$ be an SPS-labeling of a given graph $G$. If all vertices of $G$ has the same label size, say m, then $f$ is an $\ell$-uniform PS-labeling of $G$, where $m_{2}=\ell$. Next, assume that $G$ is bipartite with bipartition $\left(V_{1}, V_{2}\right)$. We can choose two positive integers $\mathrm{m}$ and $\mathrm{n}$ such that $m n=\ell$. Label all vertices in $V_{1}$ by distinct geometric progressions of size $\mathrm{m}$ and label all vertices in $V_{1}$ by distinct geometric progressions of size n so that all edges in $G$ have the same label size $\ell$. That is, $f$ is an $\ell$-uniform PS-labeling of $G$.

Conversely, let $f$ be a strongly $l$-uniform PS-labeling of $G$. If $|f(v)|=m \forall v \in V(G)$, then

$\overline{\text { Journal of Combinatorics, Information \& System Sciences }}$ 
the proof is complete. Hence, assume that not all vertices of $G$ have the same label size. Then, take an arbitrary vertex $v$ of $G$ and label it by a geometric progression of size $m$. Choose any vertex $u$ adjacent to $v$ in $V(G)$. The label size of $u$ must be $\frac{\ell}{m}$, where $m \neq \frac{l}{m}$. Since $f$ is a strongly uniform PS-labeling, every vertex $w$ adjacent to $u$ must have the label size $\mathrm{m}$ so that the label size of the edge $u w$ is $\ell$. Hence, we notice that the set-label of any vertex in $G$ is either $\mathrm{m}$ or $\frac{\ell}{m}$. Let $V_{1}$ be the set of vertices having the label size $m$ and $V_{2}$ be the set of vertices having the label size $\frac{\ell}{m}$. Clearly, $V_{1} \cup V_{2}=V(G)$. Since $m^{2} \neq \frac{\ell^{2}}{m^{2}}=\ell$, no two vertices in $V_{1}$ and no two vertices in $V_{2}$ are adjacent to each other. That is, $\left(V_{1}, V_{2}\right)$ is a bipartition of $G$, completing the proof.

Remark 2.6. In view of Theorem 2.5 , we have the following immediate observations.

(i) If $f$ is an $\ell$-uniform SPS-labeling of a graph $G$, where $\ell$ is not a perfect square, then $G$ is bipartite.

(ii) An SPS-labeling $f$ of $G$ can be an $(m, l)$-uniform PS-labeling if and only if $m^{2}=\ell$

\section{EXQUISITE PRODUCT SET-LABELING}

It is to be noted that $A$ and $B$ need not be the subsets of their product set $A * B$ in general. Hence, it is interesting to determine the conditions for the set-labels of the end vertices of an edge of a graph $G$ to be the subsets of the set-label of that edge. In view of this problem, we define:

Definition 3.1. An exquisite product set-labeling (EPS-labeling) is a PS-labeling

$f: V(G) \rightarrow P(X)$ with the induced function $f: E(G) \rightarrow P(X)$ defined by $f^{*}(u v)=f(u) *$ $f(v), u v \in E(G)$, such that $f(u), f(v) \subseteq f^{*}(u v)$ for all adjacent vertices $u, v \in V(\mathrm{G})$.

Then, a necessary and sufficient condition for a PS-labeling to be an EPS- labeling is given below.

Theorem 3.1. A PS-labeling $\mathrm{f}$ of a graph $G$ is an EPS-labeling if and only if the set-label of every vertex must contain 1 .

Proof. The proof is immediate from the fact that a set of positive integers $A \subset \mathbb{N}$ is a subset of the product set $A B$ if and only if $1 \in B$, where $\mathrm{B} \in \mathbb{N}$.

$\overline{\text { Journal of Combinatorics, Information } \& \text { System Sciences }}$ 
Figure 1 depicts a graph which has an EPS-labeling.

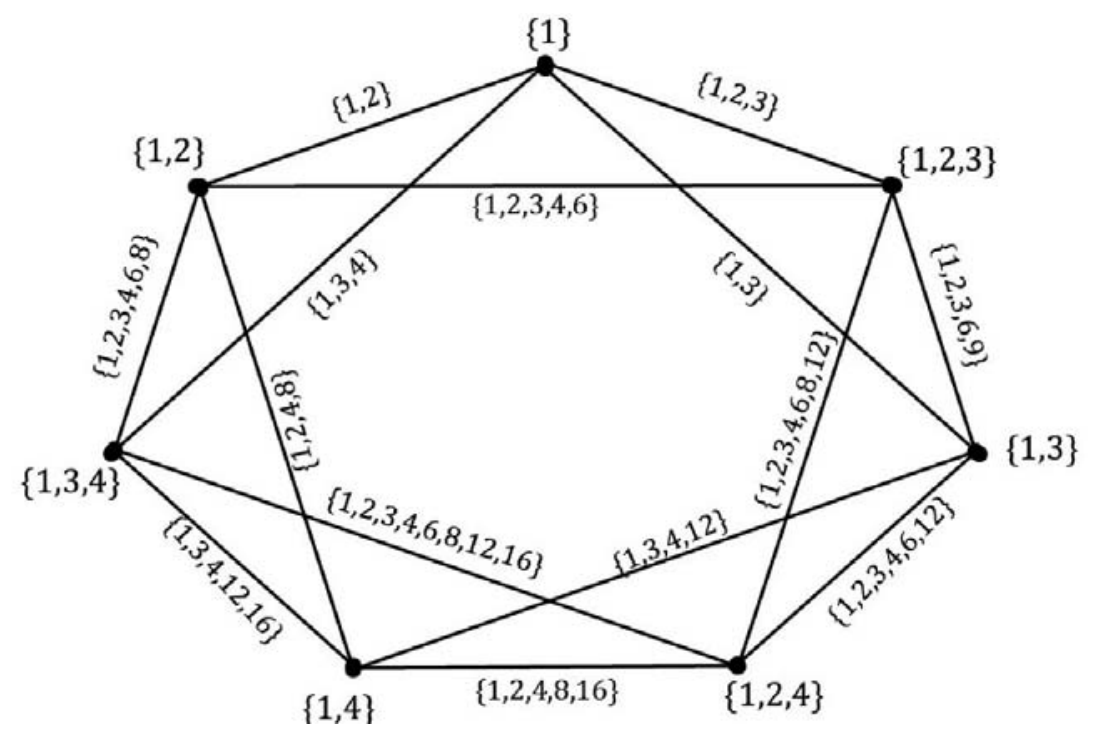

Fig. 1: An illustration to an EPS-labeled graph.

Note that the quotient-set of a set $A$ is the set defined by $Q_{A}=\left\{\frac{a}{b}: a, b \in A, a \geq \mathrm{b}\right\}$. The following result describes a necessary and sufficient condition for an EPS-labeling of $\mathrm{G}$ to be an SPS-labeling of $G$.

Theorem 3.2. An EPS-labeling $f$ of a graph $G$ is an SPS-labeling of $G$ if and only if $Q_{f(u)}$ $\cap Q_{f(v)}=\phi$ and $f(u) \cap f(v)=\{1\}$, for any two adjacent vertices $u$ and $v$ in $G$.

Proof. Let $f$ is also an EPS-labeling of $G$. First assume that $\mathrm{f}$ is an SPS-labeling of $G$. In [5] it is proved that a PS-labeling $f$ of $G$ is an SPS-labeling if and only if $Q_{f(u)} \cap Q_{f(v)}=\phi$ for all $u v \in E(G)$. Hence, it remains to show that $f(u) \cap f(v)=\{1\}$. Since $\mathrm{f}$ is an EPS-labeling, by Theorem 3.2, the set-label of every vertex must contain the element 1 . Therefore, 1 is a common element of both $f(\mathrm{u})$ and $f(v)$. If $f(u)$ and $f(v)$ contains another common element $x$, then the difference $\frac{x}{1}=x$ belongs to both $Q_{f(u)}$ and $Q_{f(v)}$, contradicting the fact that $Q_{f(u)} \cap Q_{f(v)}$ $=\phi$. Therefore, $f(u) \cap f(v)=\{1\}$.

Conversely, assume that $\mathrm{f}$ is an EPS-labeling such that $Q_{f(u)} \cap Q_{f(v)}=\phi$ and $f(u) \cap f(v)=$ $\{1\}$, for all uv $2 \mathrm{E}(\mathrm{G})$. Then, all terms of the product set $f(v) * f(v)$ will be distinct and hence

$\overline{\text { Journal of Combinatorics, Information } \& \text { System Sciences }}$ 
we have $\left|f^{*}(u v)\right|=|f(u)||f(v)|$ for all $u v \in E(G)$. Hence, $f$ is an SPS-labeling of $G$. This completes the proof.

\section{TOPOLOGICAL PRODUCT SET-LABELING OF GRAPHS}

Two sets $A$ and $B$ of positive integers are said to be the factor sets of another set $C$ of positive integers if $\mathrm{A} * B=C$.

Definition 4.1. A product set-labeling $f: V(G) \rightarrow P(X)-\{\phi\}$, defined on a graph $G$ is said to be a topological product set-labeling (TPS-Labeling) of $G$ if $f(V) \cup\{\phi\}$ is a topology on the ground set $X$.

The following theorem discusses a necessary and sufficient condition for a PS-labeling to be a TPS-labeling.

Theorem 4.1. A connected graph $G$ has a TPS-labeling if and only if $G$ has some pendant edges.

Proof. First assume that $f$ is a TPS-labeling of $G$. Then, by Definition 4.1, $f(V) \cup\{\phi\}$ is a topology on the ground set $X$. Therefore, $X \in f(V)$. We claim that $X$ can be the set-label of a pendant vertex. Note that $\{1\}$ and $X$ itself are the only factor sets of the ground set $X$. Then, $X$ can be the set-label of a vertex which can be adjacent to the vertex having set-label $\{1\}$ only and hence the vertex with set-label $X$ will be a pendant vertex of $G$. Also, if $x_{r}$ is the maximal element in $X$, then the vertices, whose set-labels contains the element $x_{r}$, can be adjacent to the vertex having set-label $\{1\}$ only. Hence, $G$ has at least one pendant vertex.

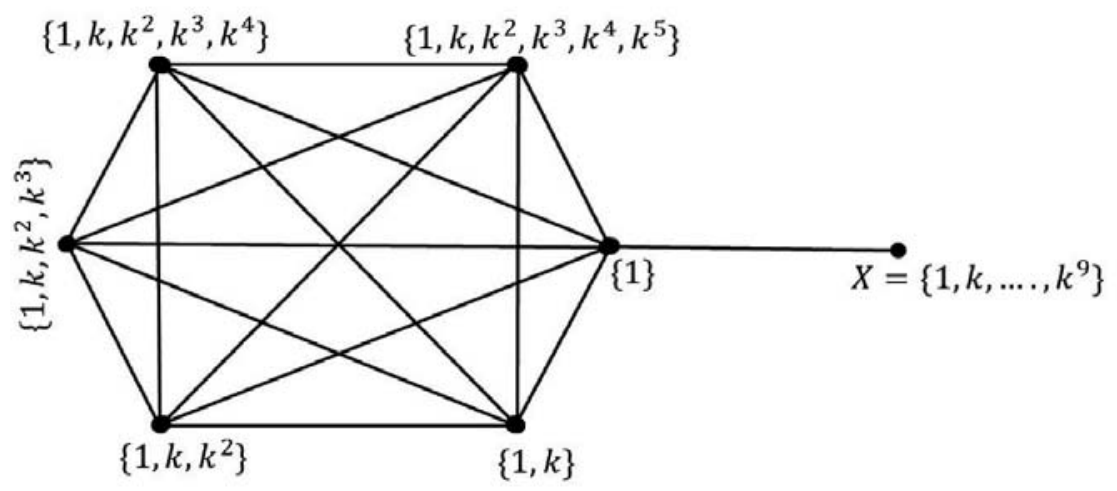

Fig. 2: An illustration to a TPS-labeled graph.

$\overline{\text { Journal of Combinatorics, Information } 83 \text { System Sciences }}$ 
Let $G$ be a graph obtained by attaching an edge from an external vertex v to an arbitrary vertex, say $v_{1}$, of a complete graph $K_{n}$. The edge $\mathrm{vv}_{1}$ is a pendant edge of $G$. Let $v_{2}, v_{3}, \ldots, v_{n}$ be the remaining vertices of $K_{n}$ in $G$. Let $X=\left\{1, k ; k^{2}, \ldots, k^{2 n-3}\right\}$ be the ground set, where $k$ is any positive integer greater than 1 . Label the vertex $v$ by the ground set $X$ itself and the vertex $v_{2}$ by the set $\{1\}$. Now, label the vertex by the set $\{1, k\}$, label $v_{3}$ by $\left\{1, k ; k^{2}\right\}$ and in general label the vertex $\mathrm{V}_{\mathrm{i}}$ by the set $\left\{1, k ; k^{2}, \ldots, \mathrm{k}^{i-1}\right\}$. (see Figure 2 for illustration). It can be seen that the collection of these vertex set-labels in $G$, together with the set $\{\phi\}$, forms a topology on $X$. Hence, the labeling mentioned above is a topological labeling of $G$.

This labeling can be extended to the graphs obtained by joining one end vertex of a path to one vertex of a complete graph (as seen in Figure 3), establishing the existence of a TPSlabeling for $G$.

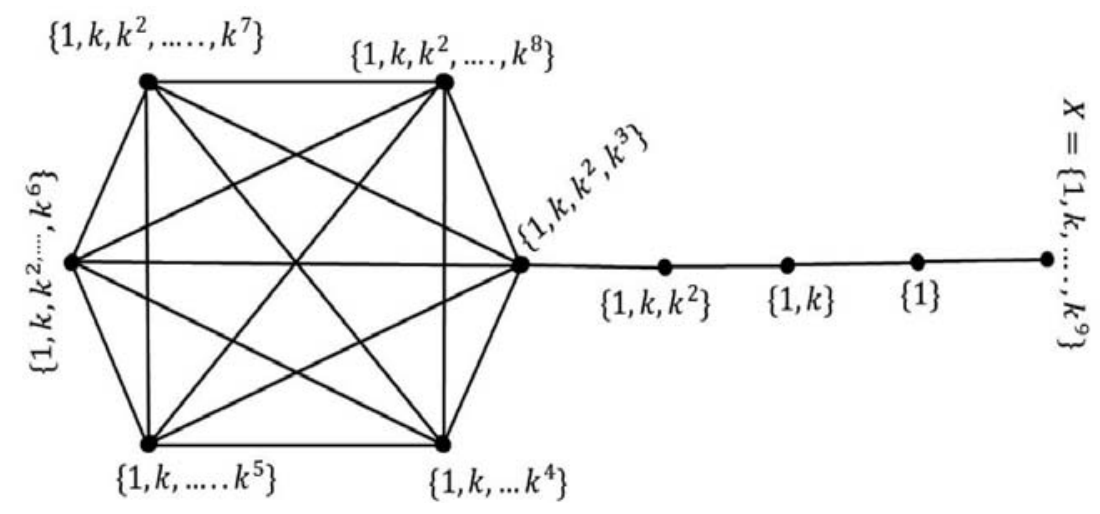

Fig. 3: An illustration to TPS-labeling of a graph

We also note that these labelings are TPS-labelings of any spanning subgraphs with exactly one pendant edge of the two graphs described above. Hence, the labeling mentioned above is a topological labeling of $G$. For the graphs having more than one pendant vertex, we can choose a pendant vertex arbitrarily for assigning the set-label $X$ and can extend this labeling procedure. This completes the proof.

\section{CONCLUSION}

In this paper, we have discussed different types of PS-labeling of graphs and the properties set-labels and the characteristics of graphs which admit these types of set-labelings. There

$\overline{\text { Journal of Combinatorics, Information } 83 \text { System Sciences }}$ 
are several open problems in this area. Some of the open problems that seem to be promising for further investigations are following.

Problem 1. Characterise the product set-labeled graphs whose vertex set-labels are geometric progressions but the edge set-labels are not.

Problem 2. Characterise the product set-labeled graphs whose edge set-labels are geometric progressions but the vertex set-labels are not.

Problem 3. Discuss the conditions required for an arbitrary PS-labeling of a graph to be a set-graceful (and set-sequential) type PS-labeling of G.

Problem 4. Discuss the conditions required for an arbitrary PS-labeling of a graph to be a bitopological type PS-labeling of $G$.

Problem 5. Discuss different types of PS-labelings for certain derived graphs such as line graphs, middle graphs and total graphs of given product set-labeled graphs.

Problem 6. Discuss the hypergraphs and signed graphs associated with the product setlabeled graph and study their properties.

Further studies on other characteristics of product set-labeled graphs corresponding to different types of PS-labelings are also interesting and challenging. All these facts highlight the scope for further studies in this area.

\section{References}

[1]. B. D. Acharya, Set-valuations and their applications, MRI Lecture notes in Applied Mathematics, No.2, The Mehta Research Institute of Mathematics and Mathematical Physics, Allahabad, 1983.

[2]. J. A. Bondy and U. S. R. Murty, Graph theory with application, North- Holland, New York, 1982.

[3]. K. A. Germina and N. K. Sudev, On weakly uniform integer additive set-indexers of graphs, Int. Math. Forum, 8(37)(2013), 1827 - 1834., DOI: 10.12988/imf.2013.310188.

[4]. F. Harary, Graph theory, New Age International, New Delhi., 2002.

[5]. S. Naduvath, Product set-labeling of certain graphs, Adv. Pure Appl. Math., 8(2)(2017), 1-8, DOI:10.1515/apam-2016-0098.

[6]. N. K. Sudev and K. A. Germina, Some new results on strong integer additive set-indexers of graphs, Discrete Math. Algorithms Appl., 7(1)(2015), 1-11., DOI: 10.1142/S1793830914500657.

$\overline{\text { Journal of Combinatorics, Information } 83 \text { System Sciences }}$ 
[7]. N. K. Sudev and K. A. Germina, A study on arithmetic integer additive set- indexers of graphs, J.Inform. Math. Sci., 109(1-2)(2018), 321-332, DOI:10.26713/jims.vl0i1+\&+2.617.

[8]. N. K. Sudev and K. A. Germina, On certain arithmetic integer additive set- indexers of graphs, Discrete Math. Algorithms Appl., 7(3)(2015), 1-15., DOI: 10.1142/S1793830915500251.

[9]. D. B. West, Introduction to graph theory, Pearson Education Inc., 2001.

\section{About the Author:}

\section{Sudev Naduvath}

Department of Mathematics

CHRIST (Deemed to be University)

Bangalore, India,

Email: sudev.nk@christuniversity.in

$\overline{\text { Journal of Combinatorics, Information } \& \text { System Sciences }}$ 